UCRL- 92022

PREPRINT

ICNF- $850310-62$

PLASMA ENGINEERING MOADES OF TANDEY MIRROR DEVICES

WITH HIGH-FIELD TEST-CELL INSERTS

M. E. Fenstermacher and R. B. Campbell

This paper was prepared for submittal to the

6th Topical Meeting on the Technology of Fusion Energy,

San Francisco, California, March 3-7, 1985

April 3, 1985
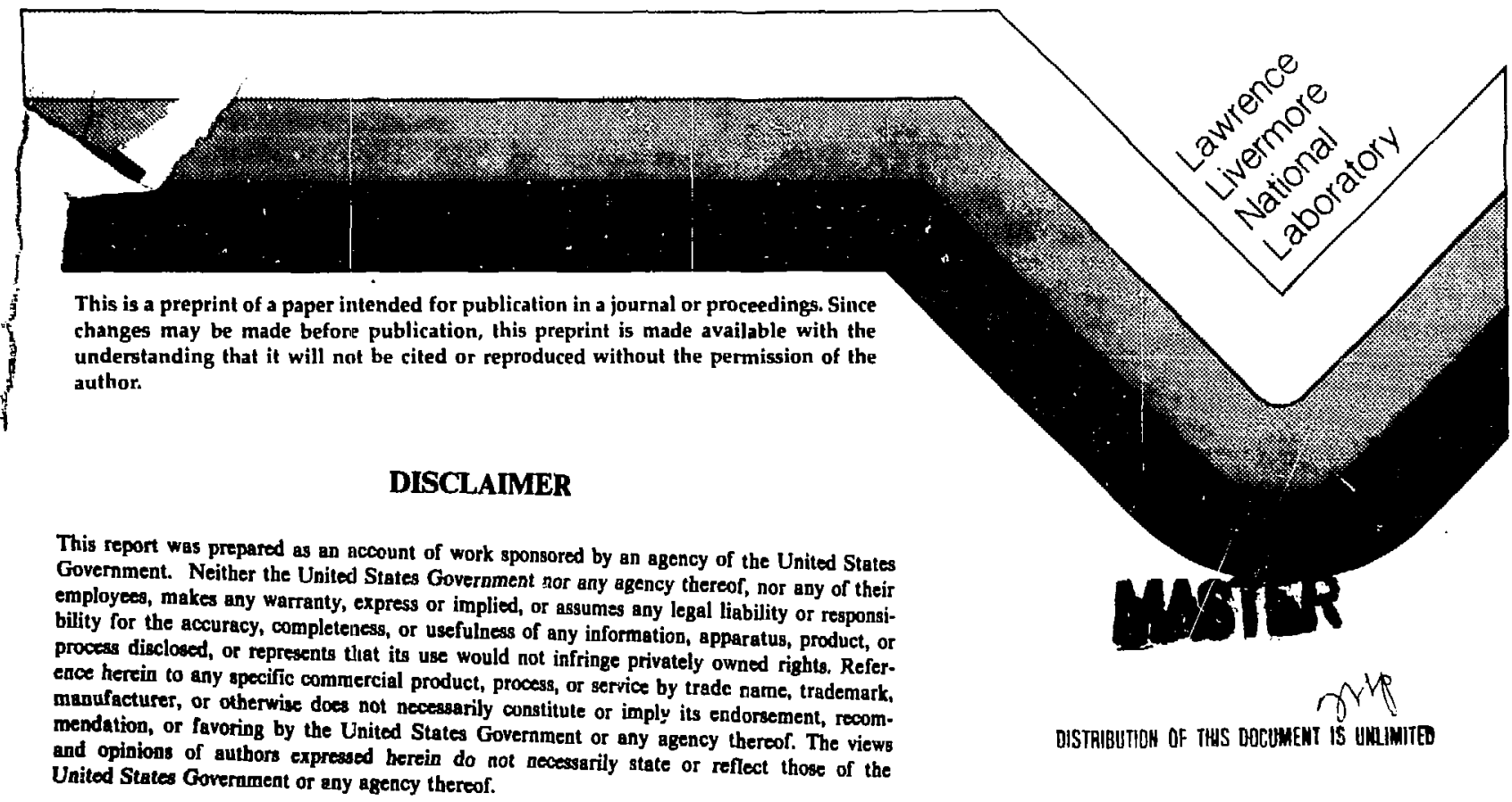

DISTRIBUTDOH OF THS DOCUMENT IS UMAMITEO 
UCRL- -92022

DE85 010046

\section{PLASMA ENGINEERING MODELS OF TANDEM MIRROR DEVICES WITH HIGH-FIELD TEST-CELL INSERTS:}

\author{
M. E. Fenotermacher ${ }^{\dagger}$ \\ TRH Inc., Rli2136 \\ One Space Park \\ Redondo Beach, CA 90278 \\ (415) 423-6308
}

\section{ABSTRAGT}

Plasma physics and engineering models of tandem mirror devices operated with a high-field technology test-cell insert in the central cell, which have been incorporated recently in the TMRBAR tandem mirror reactor physics code, are described. The models include particle and energy balance in the test-cell region as well as the interactions between the test-cell particles and those flowing through the entire device. The code calculations yield consistent operating parameters for the test-cell, central cell, and end cell systems. A benchmark case for the MFTF- $\alpha+T$ configuration is presented which shows good agreement between the code results and previous salculations.

\section{INTRODUCT ION}

The purpose of this paper is to document models of tandem mirror operation with high-field, beam-driven technology test-cell inserts, which have been developed recently at LLNL. The models have been incorporated in the TMRBAR tandem mirror reactor physics code. So far results have been obtained only for devices with MARS-type double yin-yang end cells though the test-cell models are not restricted to devices of this type.

A high-field beam-driven teat-cell inserted in the central cell of a tandem mirror device can be used to do integrated technology testing of fusion reactor components (blankets, shields, structural components etc.) in a fusion environment. Reactor like conditions (high wall

loading and fusion power density) are achieved

*Work performed under the auspices of the U.S. Departmen " of Energy by the Lawrence Livermore National Laboratory under contract number W-7405-ENG-48.

$t_{\text {On }}$ assigrment to Lawrence Livermore National Labotatory, P.0. Box 5511, L-644, Livermore CA 94550.

\author{
R. B. Campbel1 $1^{\dagger}$ \\ TRH Inc., R1/2136 \\ One Space Park \\ Redondo Beach, CA 90278 \\ (415) 423-0707
}

in the test-cell region by injecting large currents of mixed deuterium-tritium neutral beams. When test-cell operation is proposed as an upgrade of an existing tanden mirror device, it is desirable that the required modifications of the end cell systems be kept to a minimum to reduce the cost of the upgrade. In these cases, steady state is obtained by adjusti... ...e axial potential profile to reduce the axial confinement thereby providing a less cnannel for the injected beam current.

The complete list of modeling equations for test-cell configurations is given in Ref. 2 . That report also includes a users guide to the version of the TMRBAR code containing the testcell models as well as sample input and output files from code runs. This paper will contain only sumaries of the most important equations.

\section{MATHEMATICAL AYPROACH}

The TMRBAR models of tandem mirror physics have been upgraded to include test-cell physics by adding energy and particle balances of the plasma ions for a cardinal point at the midplane of the test-cell. The ion and electron balances for the remainder of the device have been modified to include test-cell effects. The testcell is treated as a separate subsystem of the device similar to the way in which the end cells are treated. The self-consistent potential for the test-cell cardinal point relative to the potential in the remainder of the central cell is calculated from a quasi-neutrality condition.

The velocity space for ions at the midplane of the test-cell is illustrated in Fig. 1 . Three classes of ions can be identified. HOT ions are those which are mirror trapped between the test-cell peak mirror fields. HOT* ions are those with pitch angle and energy such that they are not mirror trapped in the test-cell but remain trapped by the central cell choke-coil ficlds. CoLD ions are assumed to form a Maxwellian distribution in the central cell region. The HOT* ion is assumed to carry sufficient energy that if it pitch angle 


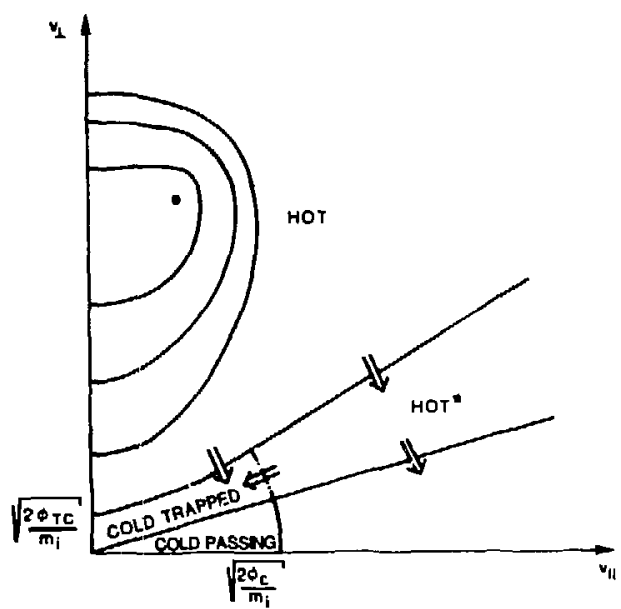

Fig. 1. Velocity space for ions at the midplane of the cest-cell.

scatters at fixed energy into the loss cone for the central cell mirror, it is not confined by the electrostatic plugging potential and is losi axially from the device.

Particle and energy balances for the HOT and HOT* ions have been modeled using modified Logan-Rensink plug models ${ }^{3}$ for beam ions injected into a magnetic trap with a potential. Pacticle flow across the boundaries in Fig. 1 and energy transfer from the hot populations to the background plasma are included. In addition, a crude model of the convection of energy due to particles crossing the boundaries in velocity space at energies higher than the COLD temperature has been developed. Finally, hot fusion alpha particles from HOT-HOT and HOT*-HOT* fuaion reactions have been included in the particle and enerzy balances.

\section{TEST-CELL MODEL EQUATIONS}

To model test-cell operation, six additional equations in six new independent variables have been added to the TMRBAR model equations. The new equations ralculate test-cell bera, potential (relative to the central cell potential), and neutron wall loading as well as describing the energy balance for the HOT particles and the particle and energy balances for the HOT particles. The independent variables used are the HOT and HOT* densities, test-cell wall loading and potential, and the HOT and HOT ${ }^{*}$ average energies.

\section{Midplanc Beta}

The equation for beta at the midplane of the teot-cell is $B_{\mathrm{m} O}=\mathrm{P} /\left(\mathrm{B}_{\mathrm{m} 0}^{2} / 2 \mu_{0}\right)$ where $\mathrm{P}$ is the total plasma pressure in the test-cel.1 and
$B_{m}$ is the vacuum magnetic field at the testcell midplane. The total pressure is the sum of pressure terms for the HOT ions, electrons, COLD ions, $H 0 i^{*}$ ions, thermal alpha particles and hot alphas. The average thermal alpha temperature is calculated as a density weighted average of the COLD ion temperature, the HOT ion average energy, and the Hot* average energy.

\section{Potential}

The potential, $\phi_{m} 0$, at the test-cell midplane relative to the central cell potential is obtained from a quasi-neutrality condition of the form $n_{e m}=n_{e c} \exp \left(\phi_{m D} / T e c\right)$ where $n_{e m}$ and $n_{e c}$ are the test-cell and central cell electron densities respectively and $T_{e}=$ is the electron temperature.

\section{Hot Average Energy}

The average energy of the $10 T$ ions which are mirror trapped in the test-cell is obtained from a Logan-Rensink plug model ${ }^{3}$ for neutral beam injection into a mirror magnetic field with a potential. The power balance in this model includes source and sink terms of the form $n^{2}(\Delta E) V /(\pi)$, where $n$ is the plasma density, $\Delta E$. the energy difference between the two particles involved, $V$ the volume, and $(n \tau)$ the appropriate particle confinement parameter. Sacrces due to hot alphas slowing down in the test-cell and a power loss rerm due to charge exchange on the test-cell neutral beams are also included. The largest power source in the test-cell comes from the neutral beam injection (proportional to $E_{I N J}-E_{L_{H} H O T}$ ) while the lergest power drain comes krom HOT ion drag on electrons (proportional to $E_{H}-3 / 2 T_{e c}$ ) where $E_{I N J}, E_{H}$, and $E_{L}, H O T$, are the neutral beam average injection energy, the HOT average energy and average lows energy, respectively. HOT ion drag on COLD ions is also an important power loss mechanism included in the test-cell models.

The confinement parameter for HOT particles in the test-cell is given by

$$
(n \tau)_{P C}=f_{F C} \frac{1.0}{(n \tau)_{i i}}+\frac{1.0}{(n T)_{e i}}-1
$$

where $(\pi))_{i}$ is the fast ion scattering time on the background ions and $(\pi \pi)_{e i}$ is the slowing down time on electrons. The coefficient $f_{P C}$ is equal to 2.0 in the standard Logan-Rensink plug model. However, this model was generated from Fokker-Planck results which assumed that the lons cone in velocity space was empty. For HOT particles in the test-cell, the loss cone is partially filled with HOT* and COLD particles. Multi-region Folker-Planck ${ }^{4}$ results indicate that for typical MFTF- $\alpha+T$ parameters the confinement parameter predicted by the otandard Logan-Rensink model may be at least $50 \%$ low. The atudies to late have used $\mathrm{f}_{\mathrm{PC}}=1.5$. 


\section{Wall Loading}

The neutron wall loading in the test-cell region is calculated as the sum of contributions from HOT-HOT fusion reactions at energy $E_{H}$, HOT*-HOT* reactions at the HOT* average energy $E *$ and COLD-COLD reactions at temperature $T_{C}$. The test-cell first wall radius is taken to be two alpha Larmor radii larger than the radius of the plasma.

\section{HOT* Particle Balance}

The density of the HOT* particles in the central cell is obtained from a particle balance which includes a source from the HOT population and loss terms inversely proportional to the total particle confinement parameter, (nT) PCC, from the Logan-Rensink model for the HOT* particles. The complete model includes the coefficient $\mathrm{C}_{\mathrm{VX}}$, HHS which gives the fraction of the HOT ions which cross the loss boundary in Fig. I into the HOT* population. It is calculated in a separate model which is described in a later section. Typically $\mathrm{C}_{\mathrm{VX}}$, HHS is in the range 0.3 to 0.5 .

\section{HOT* Average Energy}

The average energy of the HOT* population is obtained from a modified Logan-Rensink plug model similar to that used for the HOT particles. In this case, however, the relative potential is zero since the HOT*'s occupy the central cell and the injection energy and angle must be calculated to represent the particles which escape from the test-cell into the HOT* class. The power balance has the same form as the HOT ian power balance with source and oink terms proportional to $n^{2}(\Delta E) v /(n t)$ and additional source terms due to hot alpha slowing down on the HOT* particles. The largest source term is due to particles escaping the test-cell mirror and entering the HOT* population. This term has $\Delta E=E_{I N J}^{*}+\phi_{m 0}-E_{L}^{*}$, HOT where $E_{I N J}^{*}$ is the average injection energy in the HOT* Logan-Rensink model (typically about $E_{L, H O T}$ ) and $E_{L}^{*}$, HOT is the average loss energy of the HOT* ions. The largest power loss from the HoT* population is due to drag on electrons with $\Delta E=E_{H}^{*}-3 / 2 T_{e c}$. Drag of HOT* ions on COLD ions is also included. The confinement parameter for the HOT* ions is calculated in the same way as ( $n T)_{P C}$ was calculated for the HOT particles using the appropriate injection energy, effective mirror ratio, and average loss energy for the HOT*'s. Multi-region Fokker-Planck results do not give a direct calibration of the confinement parameter coefficient for the HOT* ions. Typically it nas been set equal to fpC in the studies so far.

\section{Convection Model}

Particles which cross the velocity space boundaries in Fig. I may enter the new population at an energy higher than the average energy of that population. In this way energy may be convected from one population to another. To account for this, the net particle flow rates across the velocity space boundaries must be calculated. These rates depend on the details of the gradients in the distribution function at the boundaries, which are not yet available from the multi-region Fokker-Planck studies. Models of the fractions of particles flowing from each hot distribution across the boundaries into adjacent regions of velocity space are described below. When the flow rates are available from the Focker-Planck studies they can be used to calibrate these models.

The fractions of HOT particles crossing the boundary into the HOT* and COLD trapped distributions are calculated by assuming that the HOT distribution is nearly Maxwellian centered about the injection point with average energy $E_{H}$ and density $n_{H}$. Contours of the distribution function from the Fokker-Planck results show that this is a good approximation. From this assumption the number density of particles on the boundary is calculated. Because the FokkerPlanck results also show that the slope of the distribution does not change dramatically along the boundary, the relative fractions of particles crossing into the COLD and HOT* populations at any point on the boundary are taken to be proportional to the density of particles at that point. Integrating over the boundary gives the fraction, $\mathrm{C}_{\mathrm{VX}}$, HC of the total loss from the HOT population into the COLD population.

The fraction of HOT particles crossing the boundary into the HOT* class is given by $\mathrm{C}_{\mathrm{VX}, \text { HHS }}=1.0-\mathrm{C}_{\mathrm{VX}, \mathrm{HC}}$. A similar model is used for the fraction of the total loss of HOT* particles into the loss cone and into the cold passing population.

Finally, note that the somewhat artificial distinctions made between the HOT ${ }^{\star}$ and COLD trapped populations in the TMRBAR models are necessitated by the passing density formulas which map the density of passing ions in each region of the end cell from a Maxwellian distribution in the central cell.

\section{CENTRAL CELL AND END CELL EQUATIONS}

Operation of a beam-driven testrell in the central cell of a tander mirror device changes the plasma physics paramerers in the entire machine. While the trends in test-cell performance can be predicted to a certain extent based on simple arguments (wall loading at fixed bets and test-cell confinement is proportional to trapped beam current etc.), the aspects of the tandem mirror physics which most often limit the achievable performance of a particular configuration concern the central cell particle and energy balances and the 
special end cell physics constraints. When the test-cell is to be inserted into a particular device as an upgrade, the maximum capabilities of the end cell systems can be the dominating factor limiting the achievable test-cell performance. For these reasons, modeling of the effects of test-cell operation on the remainder of the device has been done in parallel with the tegt-cell modeling. The changes in the physics models of the central cell and end cells which are required when a test-cell is operated in the device are described below.

\section{Global Charge Neutrality}

Global charge balance is achieved in the models by setting the net loss rates of ions and electrons from the device equal. In the charge balance equation used to obtain the central cell potential, $\phi_{e}$, the terms which must be modified when a test-cell is operated in the central cell are the ion burn-up and direct particle loss terms. Burn-up of tha HOT ions ia the test-cell and HOT* ions in the central cell are included. Direct loss of HOT* ions axially from the central cell is modeled in addition to direct loss of COLD ions both axially and radially. Finally, the electron loss terms are modified to take inco account the electrostatic potential, $\phi_{\operatorname{m} 0}$, in the test-cell region relative to the central cell potential.

\section{Electron Power Balance}

The electron power balance changes dramatically when a test-cell is added in a tandem mirror device. Drag of the hot ion components on the Maxwellian electron background typically produces the dominant power sources for the electrons. The drag terms are of the form $n^{2}(\Delta E) V /(n T)$. The models include COLD ion drag on electrons in the central cell with $\Delta E=1.5\left(T_{c}-T_{e c}\right)$, HоT ion drag on electrons in the test-cell with $\Delta E=E_{H}-1.5 \mathrm{~T}$ ec, warm alpha and HoT* ion drag on central cell electrons with $\Delta E=1.5\left(\tau_{\alpha, A V G}-T_{e c}\right)$ and $\Delta E=E_{H}^{*}$ - $2.5 \mathrm{~T}_{\text {ec }}$ respectively. Finally the power lost when electrons are lost from the device, which is proportional to the total energy the electron carries when it leaves the device axially, has been modified to take into account the test-cell potential $\phi_{t: 0}$.

\section{Ion Power Balance}

When a test-cell is operated in the device the COLD ion power bslance includes source terms describing energy trangfer between the hot populations and the COLD ions as well as power convected into the Maxwellian distribution by particles crossing the boundaries in velocity s pace with energy above the COLD ion temperature. In the test-cell modeling terms of the form $n^{2}(\Delta E) v /(n \tau)$ hare been added to account for HOT ion energy transfer to COLD ions in the testcell, $\Delta E=E_{H}-1.5 T_{c}$, and HOT* ions transfer- ring energy to the COLD population in the central cell and test-cell with $\Delta E=E_{H}^{t}-1.5 T_{C}$. The convection term takes the form

$n^{2}\left(E_{C}\right) V /(n \tau)_{P C}$ where $E_{C}$ is the average energy that the HOT ion carries across the boundary into the COLD distribution. Two models are available in the code, one sets $E_{c}=T_{c}$ and the other uses $E_{c}=E_{L}$ HOT $-\phi_{c} C_{V X}$, HHS where $\phi_{c}$ is the electrostatic confining potential in the plug relative to $\phi_{e}$. The latter model assumes that the average energy carried by a HOT ion intr the HOT* population is $\Phi_{C}$. A similar term descrihing the power convected into the COLD trapped population from the HOT* class is also included. The other terms in the ion power balance which include test-cell effects are described in detail in Ref. 2 .

\section{Radial Confinement Parameter}

For test-cell configurations, the radial confinement parameter, $(m) C_{R}$, has been defined directly in terms of the trapping current of COLD ions in the transition region. The assumption is made that the trapping current calculated from the Futch-Lodestro formula ${ }^{5}$ is equal to the current which is pumped out by the drift pump coils. This definition is consistent with the radial and axial power loss terms.

\section{Central Cell Density}

The central cell density is calculated from the equation for central cell beta. The allowable central cell beta input to the model depends on the test-cell beta and the relative lengths of the test-cell and central cell. In general, since the tatal MHD drive in the central cell region which can be stabilizet by the end cells is a pressure weighted normal curvature, bad curvacure regions in the testcell require that as test-cell $\beta$ increases, central cell $B$ decreases. For each calculation the $\beta$ values are fixed at a point which is MHD stable and the central cell density is calculated from

$$
n_{c}=\beta_{c} \frac{B_{C}^{2}, V A C}{2 \mu_{0}}-\left(P_{1}+P_{2}\right) / T_{5 U M}
$$

where $P_{1}$ is the sum of the pressures attributable to HOT* ions and the corresponding additional electrons required for central cell charge balance, and $P_{2}$ is the hot alpha pressure in the central cell due to HOT*-HOT* fusion reactions. The factor $\mathrm{T}_{\mathrm{SUM}}$ is the sum of the COLD ion temperature, the thermal alpha temperature weighted by the concentration of alphas, and the electron temperature.

\section{Alpha Particle Balance}

The total current of alpha particles produced in fusion reactions, including HOT-HOT and HOT*-HOT* reactions, is set equal to the Futch-Lodestro formula for the trapping current 
of alphas in the transition to yield an equation which is solved for $B_{\alpha}$ in the transition, where $E \alpha$ is the ratio of the total density of alphas to the passing density in the transition.

\section{Cold Fuel ing by Test-Cell Beams}

In test-cell configurations the neutral beam current trapped in the test-cell plasma is large. Some of these particles scatter and drag into the CólD population. The central cell fueling current requirement is calculated by the model as the total loss of central cell ions due to burn-up, radial and axial loss (including drift pumping) minus the current flowing into the COLD population from the HOT and HOT* populations. The model can yield either a positive or negative COLD fueling current requirement. Posicive $I_{F U E L}$ indicates that a supplemencary fueling system (pellet injector, low energy neutral beam system etc.) is required to achieve the steady state central cell density which has been calculated. Negative $I_{\text {FUEL }}$ indicates an unphysical imbalance of the colo ion flow rates since there are more particles entering the COLD population than are being lost. This can usually be remedied with the code model by lowering the test-cell beta or lowering the axial confinement parameter $(n \tau)_{C A}$.

\section{BENCHMARK CASE}

The test-cell models incorporated in the TNRBAR code have been benchmarked against calculations done for MFTF- $\alpha+T .6$ A detailed comparison of the results with the reference parameters is given in Ref. 7 .

For this paper a new benchmark case has been obtained using a different set of constraints than the case in Ref. 7. The new results from che code model are given in Table l. For this benchmark case the lengths, magnetic field values, beta values, and plasma radii for the entire device were the same as the reference values. In addition, the CoLD ion temperature and the test-cell average injection energy have been $f$ ixed at the reference values. (Note that in Ref. 7 the test-cell trapped current was fixed to the reference value and the average injection energy was varied to obtain energy balance.) Finally, the plug ion confining potential was veried to produce an operating point for which no supplemental fueling of the centra! cell would be required.

Comparison of the values in Ref. 6 with those in Table 1 shows that with the constraints used for this benchmark case the present model $y$ ields an operating point with higher neutron wall loading $\left(\Gamma_{\mathrm{TC}}=2.78 \mathrm{~mW} /{ }^{2}\right.$ compared with $2.0 \mathrm{MW} / \mathrm{m}^{2}$ in the reference calculation) but the required test-cell neutral beam power is also significantly higher ( $P_{N B}, T C=23.9 \mathrm{MW}$ compared with $14.4 \mathrm{MW})$. As in the benchmark calculation
Table 1. Benchmark parameters for MFTF- $\alpha+T$ bigh $\Gamma$ mode from present models.

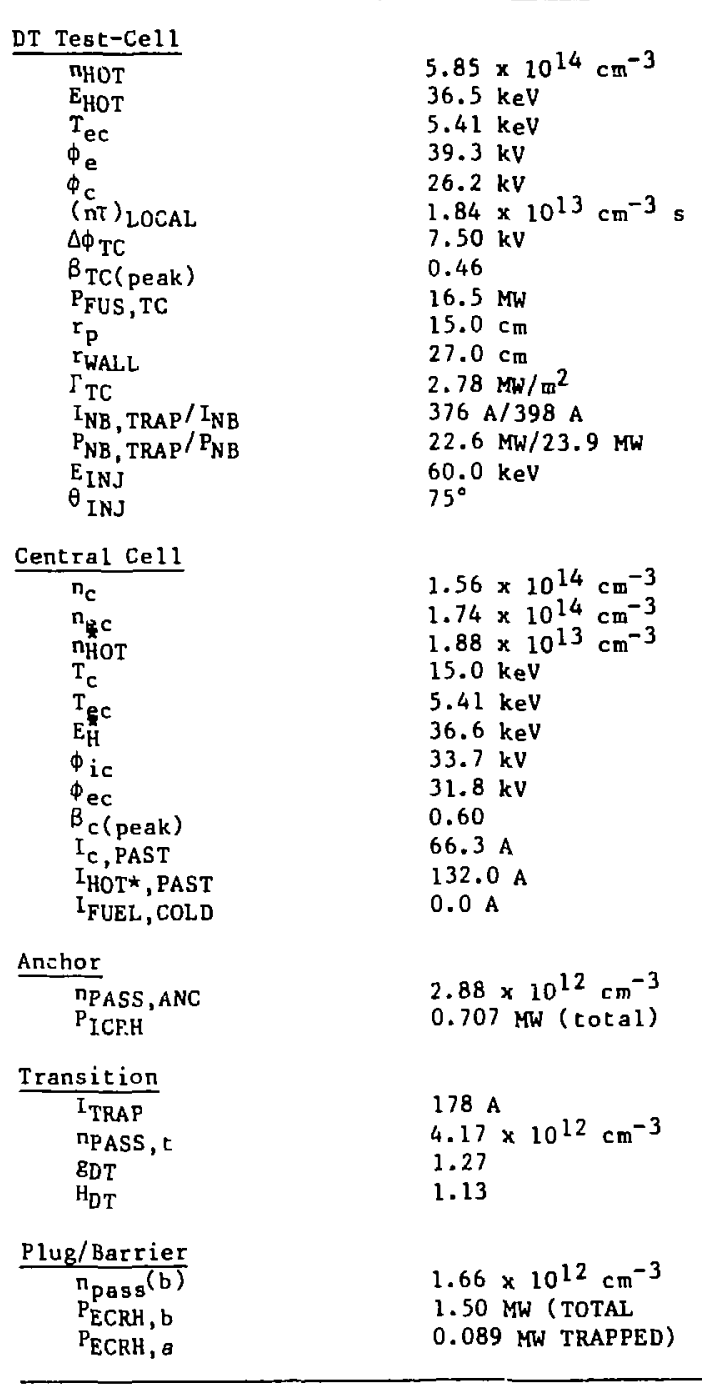

from Ref. 7, higher neutral beam power is required because direct losses of central cell particles due to pitch angle scattering from the HOT* population into the logs cone have been included in the present models. The benchmark case in Table 1 shows 132 A lost from the HOT* distribution. This combined with the $178 \mathrm{~A}$ of ions trapped in the transition region (pumped out by the drift pump system) and $66 \mathrm{~A}$ of axial loss current from the COLD population accounts for the required neutral beam trapped current in the test-cell of $376 \mathrm{~A}$. 
The drift pump current requirement is higher in the present case ( $178 \mathrm{~A}$ compared with $97 \mathrm{~A}$ ) because the $g$ value obtained with the present model (consistent with the particle, energy, and potential calculations for the device as a whole) is only 1.27. The trapping current is proportional to the product $\mathrm{K}_{\mathrm{TRAP}} \mathrm{n}_{\mathrm{PASS}, \mathrm{t}} / \mathrm{T}_{\mathrm{c}}^{\mathrm{3}} / 2$ where

$$
\mathbf{k}_{\text {TRAP }}=\mathbf{H} \frac{\mathrm{g}^{10 / 3}}{(\mathrm{~g}-1)^{7 / 3}}
$$

(see Refs, 1 and 5). Therefore, at fixed $T_{c}$, even though the present model finds a lower passing density in the transition (due to the energy cutoff described in Ref. 7), the trapping current is much larger than in the reference case because the factor $K_{T R A P}$ is much larger $\left(K_{\text {TRAP }}=53.2\right.$ compared with 4.5$)$.

Finally, the higher wall loading ia the present case results from higher $10 \mathrm{~T}$ ion density in the test-cell. This results because HOT ion drag on COLD ions has been included in the present models. This energy loss mechanism from the HOT population yields lower HOT ion average energy ( $36.5 \mathrm{keV}$ compared with $40 \mathrm{keV}$ ) than in the reference case. At fixed test-cell beta this results in higher test-cell ion density. In addition, since HOT ion irag on electrons dominates the electron powet balance the electron temperature is also lower than the reference value $(5.4 \mathrm{keV}$ compared with $7.0 \mathrm{keV})$. Since confinement parameters for the HOT particles scale with $\mathrm{T}_{\mathbf{e}}^{3 / 2}$ and the required neutral beam current to the test-cell is pcoportional to $\mathrm{n}^{2} /(\mathrm{nT})$, higher test-cell density and lower electron temperature yield much higher beam current ( $376 \mathrm{~A}$ compared with $190 \mathrm{~A})$ in the present case.

\section{CONCLUS IONS}

Models of the energy and particle balance for a high-field technology test-cell operated in the central cell of a tandem mirror have been developed. In the models the test-cell io treated as a separate subsystem with potential referenced to the central cell potential. Hot mirror trapped populations in both the testcell and central cell regions are considered. Interactions between these hot particles and the Maxwellian electrons and ions in the central cell are included. The relative potential at the midplane of the test-cell is calculated consistently from a quasi-neutrality condition.

The particle and energy balances for the HOT mircor trapped ions in the test-cell and the HOT mirror trapped ions in the central cell ate calculated separately. Both are formulated in terms of a Logan-Rensink plug model for neutral beam injection into a mirror cell with a potential. A crude model for the net flow of particles across the velocity space boundaries separating the different populations is included. Hot alpha effects due ta alphas born from HOT-HOT reactions and HOT*-HOT* reactions in the test-cell and central cell are modeled. Finally, the ion particle balance for the entire device includes fueling of the central cell Maxwellian plasma by a fraction of the ions injected into the test-cell region. In addition, the axial lose of HOT* particles from the central cell due to pitch angle scattering out of the central cell mirror trap at energies above the electroscatic confining potential is mode led.

\section{REFERENCES}

1. R. B. CAMPBELl, TMRBAR - A Code to Calculate Plasma Parameters for Tanden Mirror Reactors Operating in the MARS Mode, Lawrence Livermore National Laboratory, Livermore, CA, UCID-19875, August 1983.

2. M. E. FENSTERMACHER and R. B. CAMPBELL, Physics Modeling of Tandem Mirror Devices with High-Field Test-Cell Inserts, Lawrence Livermore National Laboratory, Livermore, CA (UCID to be published).

3. B. G. LOGAN, A. A. MIRIN, and M. E. RENSLNK, "An Analytic Model for Classical Confinement in Tandem Mirror Plugs," Nuc. Fus. 20, 1613 (298C: .

4. Y. MATSUDA and J. J. STEWART in Proc. of the 10th Conf on Numerical Solutions of Plasmas, San Diego, January 4-6, 1983 (GA Technologies Inc., San Diego, 1983) Paper 2B8.

5. A. H. FUTCH and L. L. LODESTRO, Collisional Trapping Rates for Ions in a Magnetic and Potential We11, Lawrence Livermore National Laboratory, Livermore, CA, UCRL-87299 (Feb. 1982).

6. K. I. THOMASSEN and J. N. DOGGETT, Eds., Options to Upgrade the Mirror Fusion Test Facility, Lawrence Livermore National Laboratory, I, ivermore, CA, UCID-19743. April 1983 .

7. M. E. FENSTERMACHER and R. B. CAMPBELL, Modeling and Optimization of MFTF- $\alpha+T$ High- $\Gamma$ Morle Performance, Lawrence Livermore National Laboratory, Livermore, CA, UCID-20284, December 1984. 